\title{
Preliminary scale-resolving simulations of laminar-to-turbulent transition in swept-wing flow
}

\author{
Marie Denison*, Anirban Garai ${ }^{\dagger}$, and Scott Murman \\ NASA Ames Research Center, Moffett Field, CA 94035
}

\begin{abstract}
Swept wings and control surfaces are common elements of modern aircraft, and it has been shown both experimentally and theoretically that laminar-to-turbulent transition of the three-dimensional boundary layer that develops over them is highly sensitive to surface roughness. Numerous studies have been conducted on the effect of discrete roughness elements or distributed roughness elements on swept flow transition, however so far limited computational effort has been dedicated to the study of transition over swept wings with randomly distributed micron-sized roughness. In the present work, we set up to reproduce the extensive experimental data base generated by Dagenhart et al [1] for the infinite swept wing NLF(2)-0415. To this purpose, we perform scale-resolving simulations of flow transition over smooth and rough surfaces using a high-order space-time spectral-element Discontinuous-Galerkin solver. Different types of surface roughnesses are implemented by elastically deforming the original mesh. The study shows that the experimental results cannot be accounted for by a perfectly smooth wing and reveals a strong sensitivity of the transition process to the representation of the surface roughness. The crossflow patterns and transition location approach those measured for some of the surface profiles, however a correlation between the wavenumber spectrum of the surface, grid resolution and boundary layer stability is yet to be established.
\end{abstract}

\section{Introduction}

The lack of reliability of current models to predict laminar-to-turbulent transition over the flight envelop of aircraft is still limiting the extent to which design can rely on computational tools, while making costly wind tunnel and flight tests a necessity [2]. Prediction of transition on practical three-dimensional wing geometries using the $e^{N}$ method coupled with laminar boundary layer and linear stability codes [3, 4] or local statistical correlation CFD models such as the $\gamma-\operatorname{Re}_{\theta}$ Langtry-Menter model [5] have proven challenging [6] -8], whereas transitional hybrid RANS-LES solvers have shown encouraging but still mitigated results [9-11]. The crossflow models implemented in RANS solvers [12, 13, 15, 16] account for the effect of roughness in a somewhat rudimentary way. For example the popular crossflow extension of the Langtry-Menter model [15] incorporates the effect of surface roughness on the transition momentum thickness Reynolds number through a correlation to experimental data gathered by Ref. [17] Radeztsky et al. for the NLF(2)-0415 swept wing for different surface finishes [17] augmented by linear stability analysis, and by Kreplin for the inclined prolate spheroid [18]. In his experiments, Radeztsky observed that a paint roughness with $9 \mu \mathrm{m}$ profile height could increase the transition Reynolds number by $70 \%$, compared to a highly polished surface with $0.25 \mu \mathrm{m}$ roughness height. In contrast, Reibert et al. observed an insensitivity of transition to an artificial distributed roughness array with an amplitude of $6 \mu \mathrm{m}$ or greater applied near the leading edge, as a result of premature nonlinear amplitude saturation of narrow-banded modes [19].

The capability to quantitatively predict the different stages of flow transition on a swept wing with realistic broad band micron-sized surface roughness (as opposed to model problems with roughness designed to excite selected modes) from the onset of crossflow instability to secondary instabilities and three-dimensional turbulent breakdown has not been demonstrated yet. Although computationally expensive, scale-resolving simulations provide insights on the mechanisms driving transition and validation data bases that can be used for transition model development. In the present work, scale-resolving simulations of laminar-turbulent transition over an infinite swept wing are performed and validated

\footnotetext{
*NASA Advanced Supercomputing Division, marie.f.denison@ nasa.gov.

†Science and Technology Corporation, anirban.garai@nasa.gov.

†NASA Advanced Supercomputing Division, scott.m.murman@nasa.gov.
} 
against the experiments conducted by Dagenhart et al. on a $45^{\circ}$ swept NLF(2)-0415 wing [1]. We investigate the ability of different surface patterns to capture the dominant crossflow modes and transition location. In the next section, we describe the spectral-element methods used to carry out the simulations. Section III outlines the NLF(2)-0415 experiments and simulation set-up, including the definition of the investigated surface roughnesses. The results obtained for the smooth and rough wings are discussed in Section IV. Finally, concluding remarks are provided in Section V.

\section{Numerical methods}

The compressible Navier-Stokes equations are solved using a high-order space-time spectral-element discontinuousGalerkin (DG) method with an entropy stable formulation [20-22]. It utilizes numerical methods and linear algebra kernels that were optimized to exploit current and projected HPC capabilities, making the code highly efficient and scalable [21, 23, 24]. The entropy-stable scheme of Ismail and Roe [25] is used for the inviscid fluxes, whereas viscous fluxes are computed using an interior penalty method with parameter based on Bassi and Rebay [26]. The non-linear system for each space-time slab is solved using a preconditioned Jacobian-free approximate Newton-Krylov solver. Integrals are approximated by the Gauss quadrature rule using twice as many quadrature points as solution points to reduce aliasing errors and increase stability when evaluating non-linear products. Variable spatial order can be specified over the domain [27]. The solver was validated up to $16^{\text {th }}$ spacial and temporal order with highly separated turbulent flows [28, 29]. Surface roughness is generated using the methodology proposed in Ref. [24, 29], wherein a representative roughness pattern is projected on to higher-order polynomials at the wall boundary and a high-order Continuous-Galerkin approach is used to perturb the volume nodes using a linear-elasticity analogy.

\section{Problem setup}

The $45^{\circ}$ swept NLF(2)-0415 wing measured by Dagenhart et al at the Arizona State University Unsteady Wind Tunnel are used as our test case [1]. In the test section special liners were installed at the ends of the wing to emulate the path of streamlines in free air over a wing of infinite span. The experiments included naphthalene sublimation and liquid-crystal flow vizualization studies to determine the extent of laminar flow and the stationary vortex wavelengths. Velocity profiles were measured with hot-wire anemometers at several spanwise stations across selected vortex tracks. Velocity profiles at the various spanwise locations and velocity contours across the vortex wavelength for both the mean and the disturbance velocities were compiled. Most of the measurements were taken at the streamwise chord based Reynods number $\operatorname{Re}=2.37 \times 10^{6}$ at the angle of attack $\alpha=-4^{\circ}$. For that reason these particular conditions were selected for our study. The simulated Mach was set to 0.1, close to the maximum wind tunnel velocity of $36 \mathrm{~ms}^{-1}$. The upper velocity range was selected because we use a compressible solver without low-Mach corrections. Figure 1 shows the wing geometry and reference axes used here, and Table 1 summarizes the key geometry and flow parameters.

Table 1 Geometry and flow conditions

\begin{tabular}{ll}
\hline Feature & Value \\
\hline Airfoil & $\mathrm{NLF}(2)-0514$ \\
Sweep angle $\Lambda$ & $45^{\circ}$ \\
Experimental Streamwise chord length $\mathrm{C}$ & $1.83 \mathrm{~m}$ \\
Reynolds number $\operatorname{Re}_{\mathrm{C}}=\frac{\mathrm{u}_{\infty} \mathrm{C}}{v_{\infty}}$ & $2.37 \times 10^{6}$ \\
Mach number $\mathrm{M}_{\text {ref }}$ & 0.1 \\
Angle of attack $\alpha$ & $-4^{\circ}$ \\
Temperature & $288.15 \mathrm{~K}$ \\
\hline
\end{tabular}

The current study uses an isoparametric mapping to generate the unstructured high-order mesh required for the fluid solver. This mapping is constructed using a zonal approach to tailor the resolution to the different physics encountered (Figs. 2 3). This zonal approach is further tailored by choosing the order of accuracy within each region, i.e. using low-order elements (fewer degrees of freedom) away from the body to the farfield (located at 50 chord lengths), and high-order elements (increased resolution) in the boundary layer and transition region. This topology is maintained in the roughness simulations, with the roughness pattern being applied to the surface, and then propagated through the 


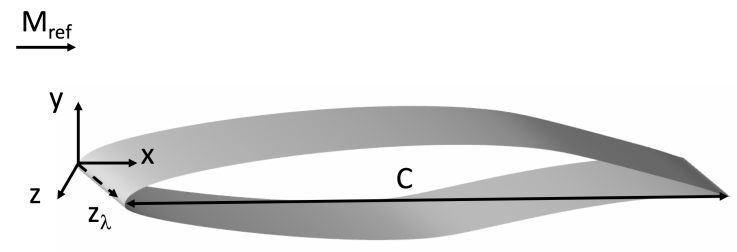

(a) Front view

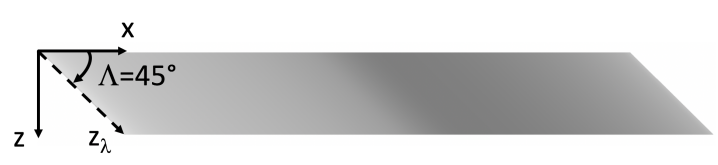

(b) Top view

Fig. 1 NLF(2)-0415 swept wing.

volume using the nonlinear elasticity solver described in the previous Section.

A multi-step approach was taken for the transition study, starting with a smooth wing with spanwise width of $12 \%$ chord length and a coarse grid to gain initial information about the flow field. Eighth-order elements were used throughout the domain (Fig. 3 (a)). Next, the span width was reduced to one stationary crossflow wavelength to limit the size of the mesh while increasing the boundary layer resolution. A graded boundary layer grid following the boundary layer profile was created, with a minimum of 40 degrees of freedom (DOF) across the boundary layer and turbulent boundary layer wall spacing of the order of the viscous lengthscale. In order to focus the resolution where it was most needed, shells of variable spatial order were defined around the wing, see Fig. 2(b) and Fig. 3 b). This grid was then deformed by means of the elastic solver to generate a first roughness pattern (see Section III). Because of convergence issues encountered with the high cell aspect ratio in the $8^{\text {th }}$ order regions around the front part of the wing, a new grid was generated with 16 instead of 40 DOF in the laminar boundary layer (Fig. 3(c)). The last grid shown in Fig. 3(d) features a larger cell count at the nose surface to improve the roughness resolution, while dropping solution order closer in the far- and mid-field. Although the grid has been only been run up to $4^{\text {th }}$ order so far, it is planned to be transferred to $8^{\text {th }}$ order in the near future. Further information on the grids is provided in Table 2

Table 2 Simulation cases

\begin{tabular}{llllll}
\hline Case & $\begin{array}{l}\text { Span width } \\
\text { S/C }\end{array}$ & $\begin{array}{l}\# O(8) \\
\text { elements }\end{array}$ & $\begin{array}{l}\# O(4) \\
\text { elements }\end{array}$ & \# DOF & Roughness amplitude $\eta / \mathrm{C}$ \\
\hline Smooth \#1 & 0.12 & $49.1 \times 10^{3}$ & 0 & $25.1 \times 10^{6}$ & n/a \\
Smooth \#2 & $\lambda_{\mathrm{z}}$ & $19.4 \times 10^{3}$ & $64.2 \times 10^{3}$ & $14.8 \times 10^{6}$ & n/a \\
Rough \#1 & $\lambda_{\mathrm{z}}$ & $19.4 \times 10^{3}$ & $64.2 \times 10^{3}$ & $14.8 \times 10^{6}$ & $\eta / \mathrm{C}=\mathrm{y}_{\text {rms,exp }} / 3 ., \eta>=0$ \\
Rough \#2 & $\lambda_{\mathrm{z}}$ & $16.2 \times 10^{3}$ & $57.6 \times 10^{3}$ & $12.7 \times 10^{6}$ & $\eta / \mathrm{C}=\mathrm{y}_{\mathrm{rms}, \exp ,}, \eta=0$ \\
Rough \#3 & $\lambda_{\mathrm{z}}$ & 0 (for now) & $28.7 \times 10^{3}$ & $3.9 \times 10^{6}$ & $\eta / \mathrm{C}=\mathrm{y}_{\mathrm{rms}, \operatorname{exo}, \eta_{\mathrm{av}}=0}$ \\
\hline
\end{tabular}

\section{A. Surface roughness}

Two types of roughness profiles were used over the front of the wing: the first is based on pseudo-random displacements with RMS amplitude corresponding to the profile of a paint applied to the NLF(2)-0514 wing reported in Ref. [17]. The node displacement along the normal to the surface pointing into the fluid domain is always positive. The roughness is applied on the upper and lower sides of the wing for a curvilinear coordinate in the interval $\mathrm{s} / \mathrm{C} \in[0,0.3]$ and it is damped to zero within the interval $\mathrm{s} / \mathrm{C} \in[0.3,0.4]$ using a shifted half-cosine function. This roughness type was used in the roughness cases \# 1 and \# 2 of Table 2 with two different amplitudes $\eta / \mathrm{C}=1 / 3 \mathrm{y}_{\mathrm{RMS} \text {,exp }}$ and $\eta / \mathrm{C}=\mathrm{y}_{\mathrm{RMS} \text {,exp }}$ (where $\mathrm{y}_{\mathrm{RMS}}$ is the RMS amplitude of the experimental profile), respectively.

The second type of roughness profile implemented in case \#3 utilizes a random distribution of normal displacements filtered by the auto-covariance length of the experimental paint profile, with RMS amplitude matching that of the experiments. An example of such profile is shown in Fig. 4. Two spanwise and streamwise periodic patches with aspect ratio 1:4 were created (one for either side of the wing), and stored in a file from which the applied grid displacements 


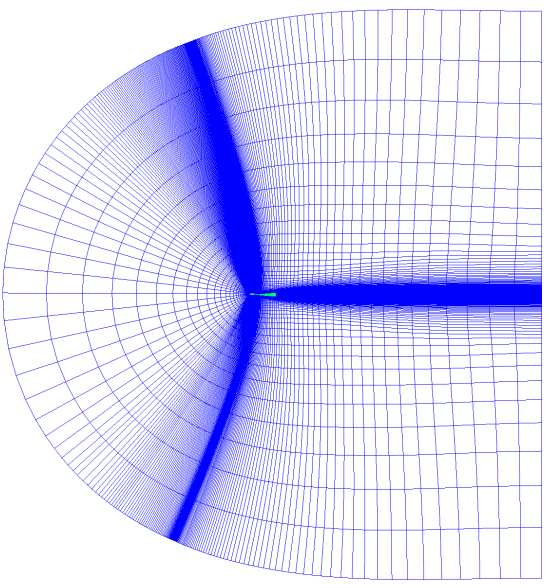

(a) Domain overview

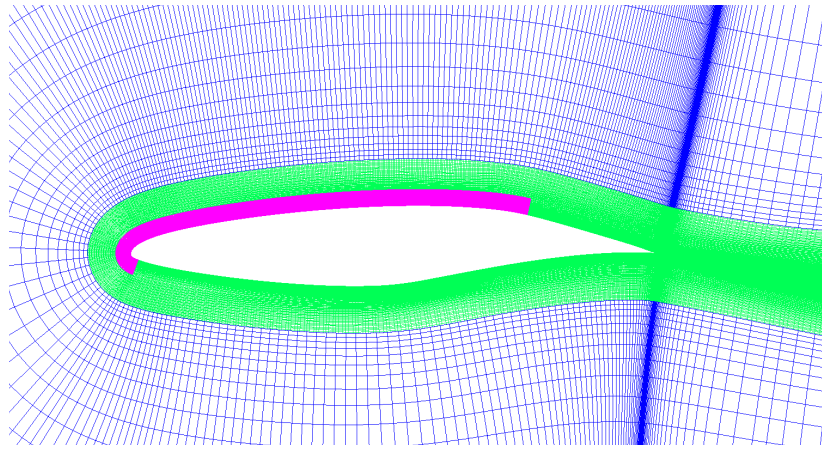

(b) Zoom on area of the wing

Fig. 2 Grid for the Smooth \#2 case colored by element spatial order (blue: $2^{\text {nd }}$ order, green: $4^{\text {th }}$ order, magenta: $8^{\text {th }}$ order).

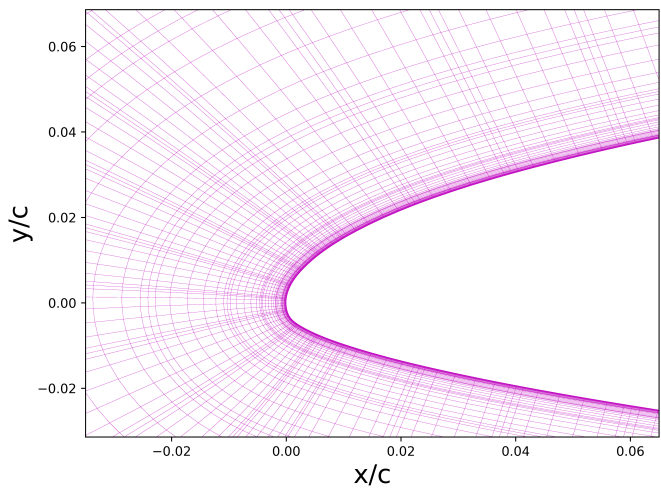

(a) Smooth \#1

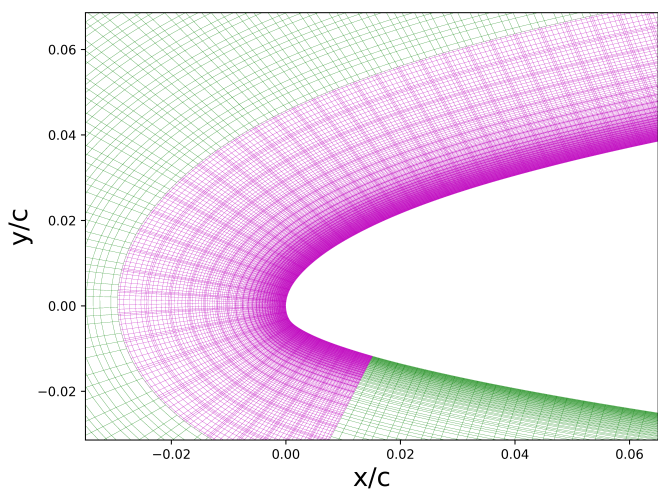

(c) Rough \#2

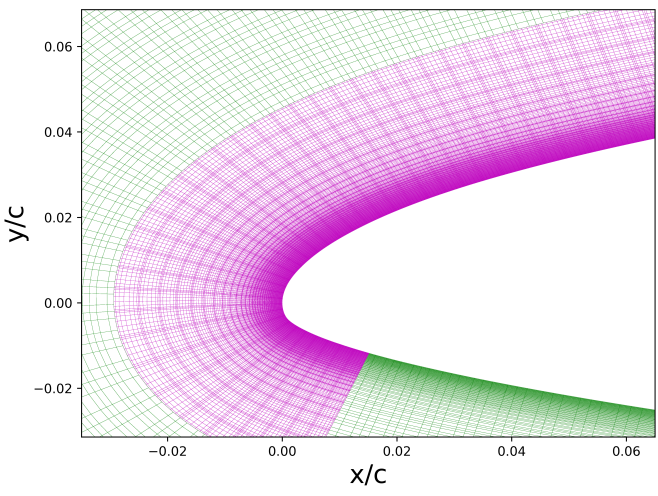

(b) Smooth \#1 and Rough \#1

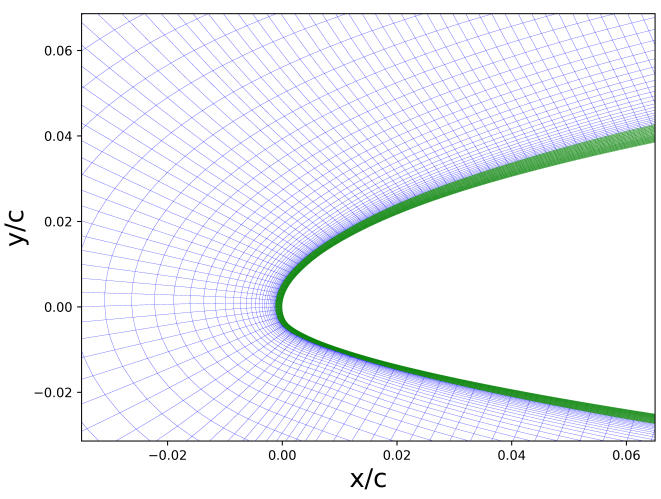

(d) Rough \#3

Fig. 3 Close-up of the grids around the leading edge of the wing colored by element order (blue: $2^{\text {nd }}$ order, green: $4^{\text {th }}$ order, magenta: $8^{\text {th }}$ order). 
were interpolated. The displacement amplitude applied to the mesh surface was adjusted to match the experimental RMS. Fig. 5 shows streamwise profiles and their discrete Fourier spectrum, whose discontinuous profile results from the limited sample size.

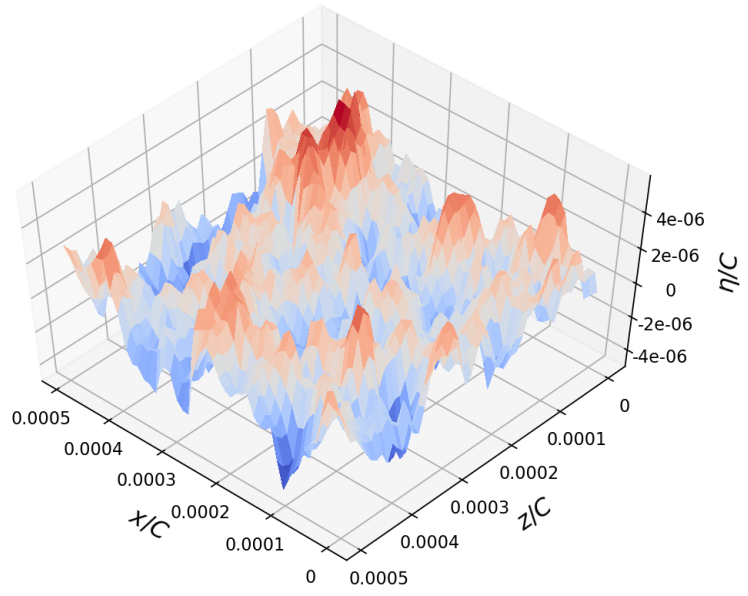

Fig. 4 Close-up of an area of the roughness patch use to deform the the rough grid \# 3.

\section{Results}

\section{A. Smooth wing}

In the experiment, the transition location was determined from the abrupt shift in sublimation rate of the naphthalene coating due to turbulence-induced shear stress increase. Transition occured at an average of $x / c=0.58$ with a series of overlapping turbulent wedges forming a saw-tooth pattern (Fig. 6(a)). In the simulation, we identify the location of transition at the middle of the accompanying friction ramp. In the case of the smooth wing \#1, transition occurs at $x / C=0.82$, after flow separation on the pressure recovery of the side of the wing, Fig. 7 . Weak crossflow vortices that are nearly parallel to the inviscid flow are observed prior to separation, and the turbulent transition front is uniform in the spanwise direction (Fig. 8). For the same flow conditions, the simulations results look closer to the $R e=1.93 \times 10^{6}$ case, Fig. 6 . b). In that image, one can distinguish the regularly spaced streaks from the crossflow vortices.

The simulation results agree well with the measurements in the laminar boundary layer over the first quarter of the upper surface (Fig. 9.a)). Further downstream, the crossflow develops in the form of skewed, counter-rotating vortices that visibly distort the mean flow, Fig. 9(b). Such distorsions cannot be distinguished in the simulated mean velocity profile, however a pattern of crossflow vortices does exist, with an amplitude that is over three orders of magnitude lower than in the experiments (Fig. 10p. The crossflow wavelength projected in the direction of the leading edge sweep $\lambda / \mathrm{c}=5 \mathrm{~mm}$ is smaller than the measured value of $6.2 \mathrm{~mm}$. The crossflow vortices are oriented at $12^{\circ}$ with respect to the freestream direction (Fig. ??), compared with $5^{\circ}$ in the experiments. The refined grid for the smooth case \# 2 yields similar results in terms of transition location (Fig. 77) and crossflow amplitude (Fig. 11), indicating that other sources of perturbation such as surface roughness or freestream disturbances are required to excite crossflow vortex growth.

\section{B. Rough surface}

Figure 12 shows the time-averaged friction coefficient for the the rough cases. Although the flow has reached a stationary state, limited statistics were available for averaging at the time of publication, which explains the oscillations of friction beyond the point of transition. The data indicate very high sensitivity of the transition to the roughness profile and mesh. The first roughness type advances the transition front upstream, closer to the experimental location of $\mathrm{x} / \mathrm{C}=0.58$. With 


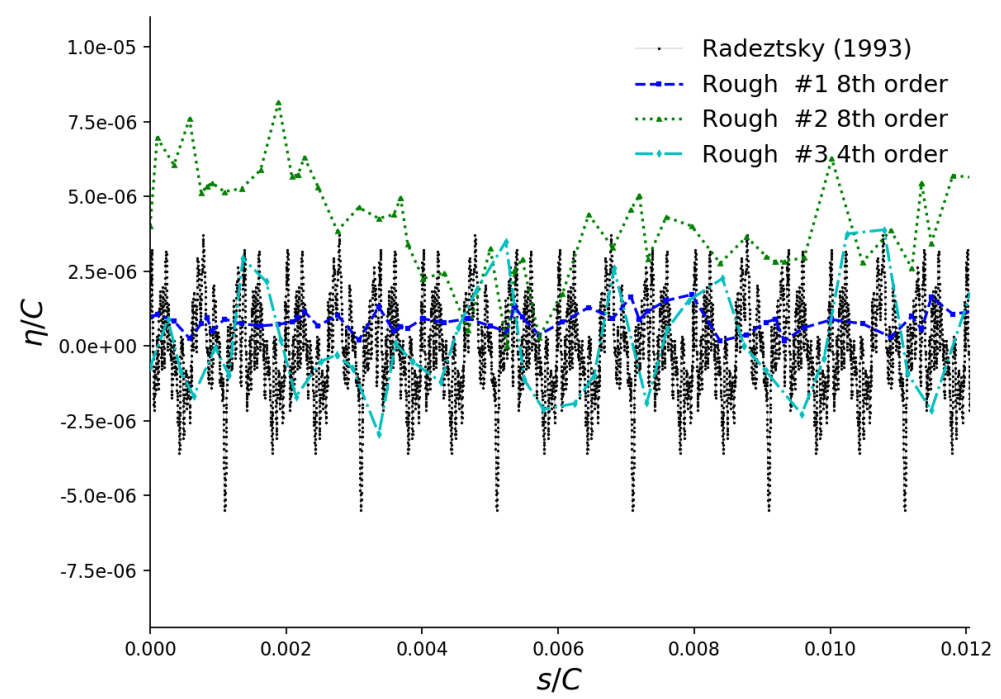

(a) Roughness profile versus curvilinar coordinate $\mathrm{s} / \mathrm{C}$ along the upper surface of the wing near the nose $(z=0)$.

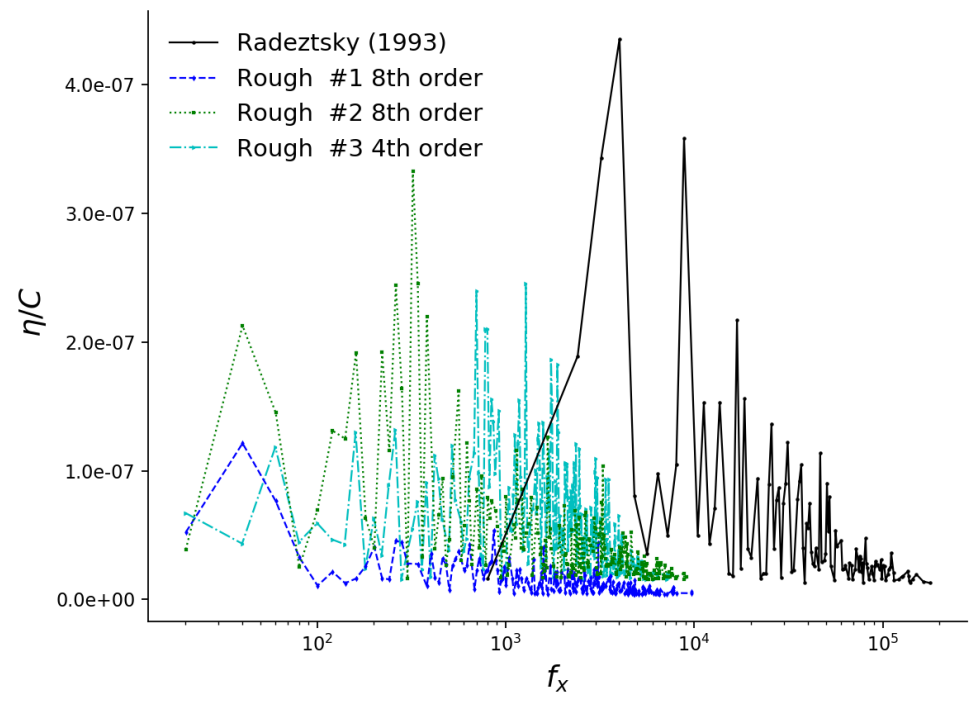

(b) Frequency

Fig. 5 Roughness profiles and their Fourier spectrum.

roughness \#2, transition is still taking place after flow separation. The third roughness results in premature transition at $\mathrm{x} / \mathrm{c}=0.17$.

Figure 13 shows profiles of instantaneous friction coefficients at $z=0$ for the smooth case \#2 and the first two roughness cases. The roughness causes small but visible friction perturbations at the leading edge of the wing. The larger oscillations in the mid section of the wing are caused by the crossflow vortices, Figure 15. As Fig. 14]indicates, the crossflow disturbances around mid-chord are in the range of those measured. They die down for roughness \# 2, and are amplified up to turbulent transition for the roughness case \#1. Small waves are riding over the stationary vortices as indicated by the red arrow in Fig. 16 The dimensional frequency of these waves is in the range of those reported by 


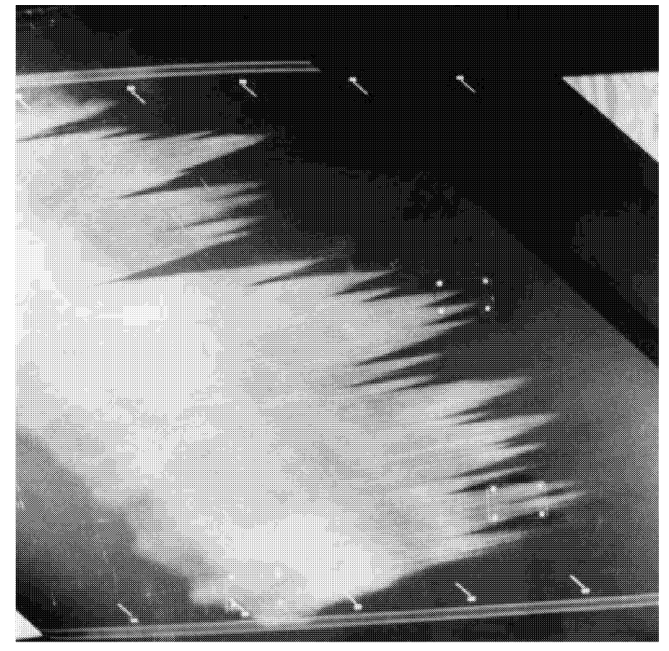

(a) $\mathrm{Re}=2.4 \times 10^{6}$

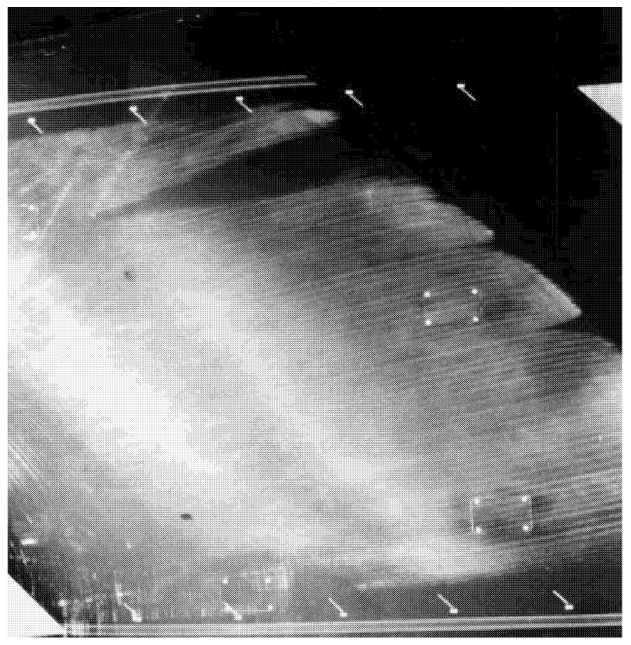

(b) $\left[R e=1.93 \times 10^{6}\right.$

Fig. 6 Naphtalene flow visualization at $\alpha=-4^{\circ}[1]$.

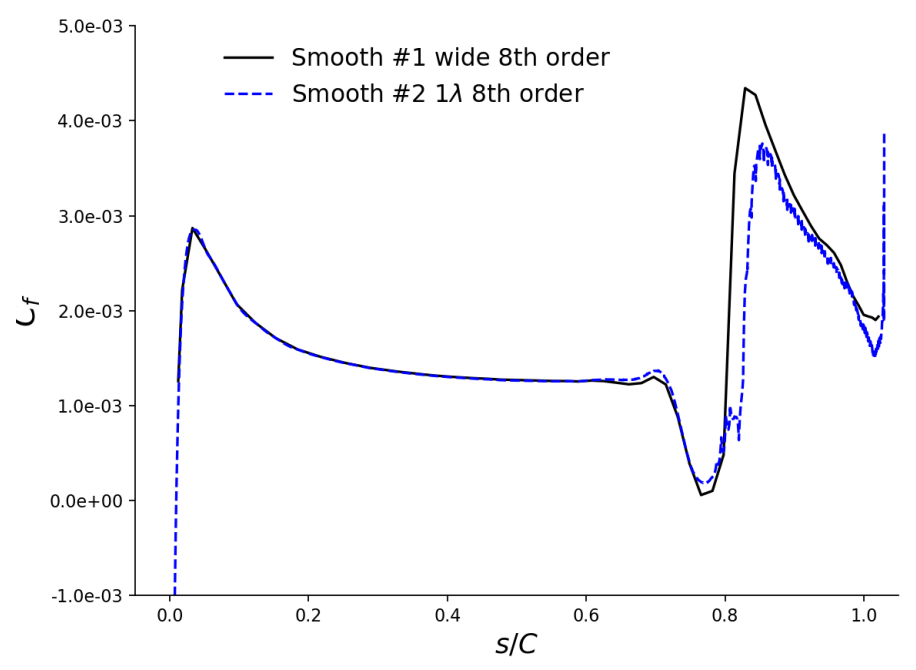

Fig. 7 Friction coefficient on the upper side of the smooth wings.

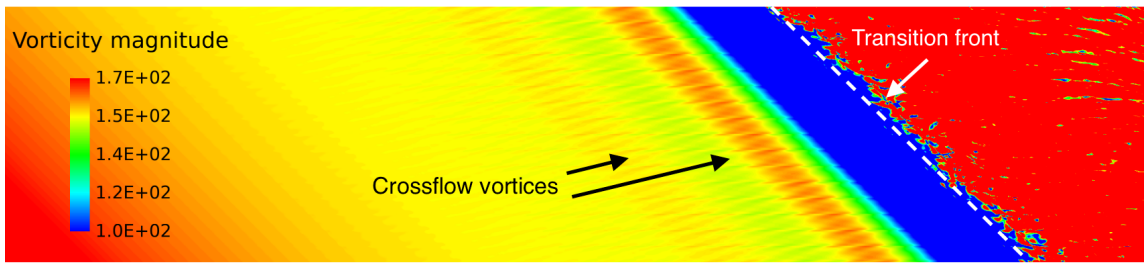

Fig. 8 Vorticity magnitude on the top side of the wing for case \#1 with smooth surface.

Dagenhart who observed $100 \mathrm{~Hz}$ traveling modes just ahead of the transition location, with an amplitude of up to $7 \%$ of the edge velocity. For the third roughness case, the wavelength of the crossflow vortices preceding transition is three times smaller than in the earlier two cases, Figure 17. To test the convective nature of the instability responsible for transition, the roughness was switched off and the simulation restarted. The temporal evolution of the friction coefficient is shown in Fig. 16. After moving upstream, which we interprete as a result of the numerical disturbance caused by the 


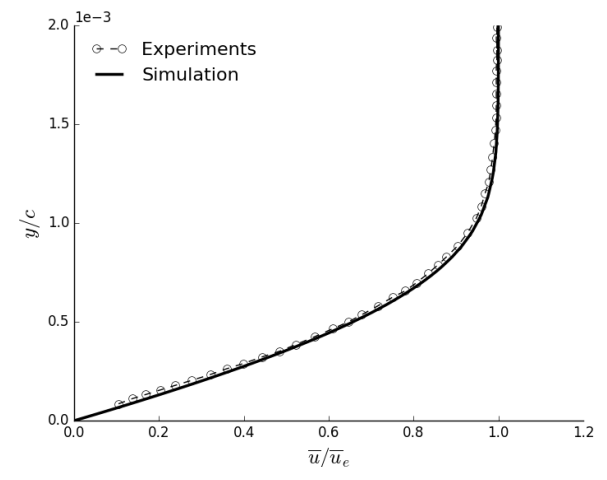

(a) $x / c=0.25$

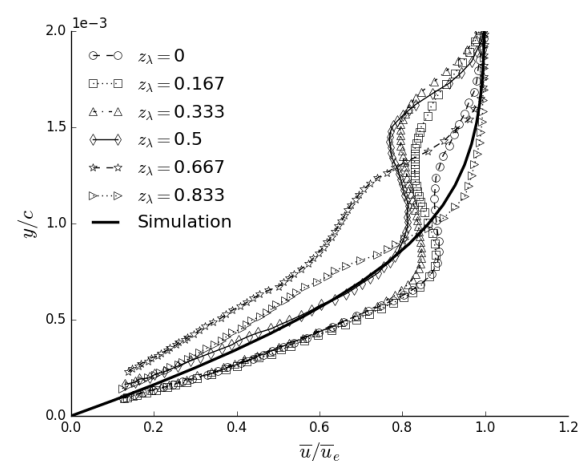

(b) $x / c=0.55$

Fig. 9 Laminar streamwise velocity profile compared to experiments (symbols) [1].

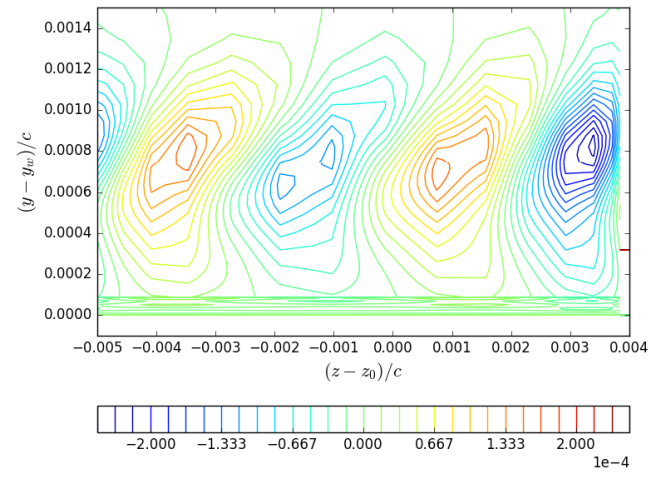

(a) Smooth case \#1

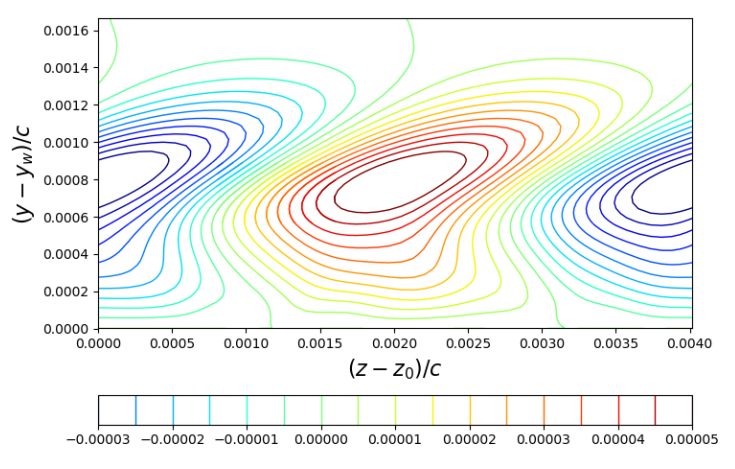

(b) Smooth case \#2

Fig. 10 Contours of streamwise velocity perturbation in a plane parallel to the wing leading edge passing through $x / C=0.55, z / C=0$.

removal of the roughness, the transition front moves back downstream and after 1.4 flow-through time it still has not moved beyond $\mathrm{x} / \mathrm{C}=0.2$. The time required to relax to the smooth case may be very long in relation with the low viscosity of the flow, or there may be some sustaining effect at play, as will be further investigated.

\section{Conclusions}

Preliminary scale-resolving simulations of flow transition over smooth and rough surfaces of the infinite swept wing NLF(2)-0415 were conducted. The simulation results for the smooth wing with large span were consistent with those obtained for one-wavelength domain and higher mesh refinement, but could not account for the experimental results. A strong sensitivity of the transition process to the roughness profiles was found. The roughness profile with the smallest amplitude yielded the results closest to the experiments in terms of the crossflow pattern, amplitude and transition location. The profile with larger amplitude and smaller vertical resolution yielded similar crossflow but was unable to move the transition location upstream. The last roughness case caused premature transition close to the nose and was preceded by higher frequency crossflow vortices. The results gathered in this work will be analyzed next in terms of the wavenumber spectrum, grids and boundary layer stability.

\section{Acknowledgments}

The authors thank the NASA Transformative Tools and Technologies (T3) Project for funding this effort, in particular Mike Rogers at NASA Ames for his support. We thank the NASA Advanced Supercomputing Division (NAS) for making 


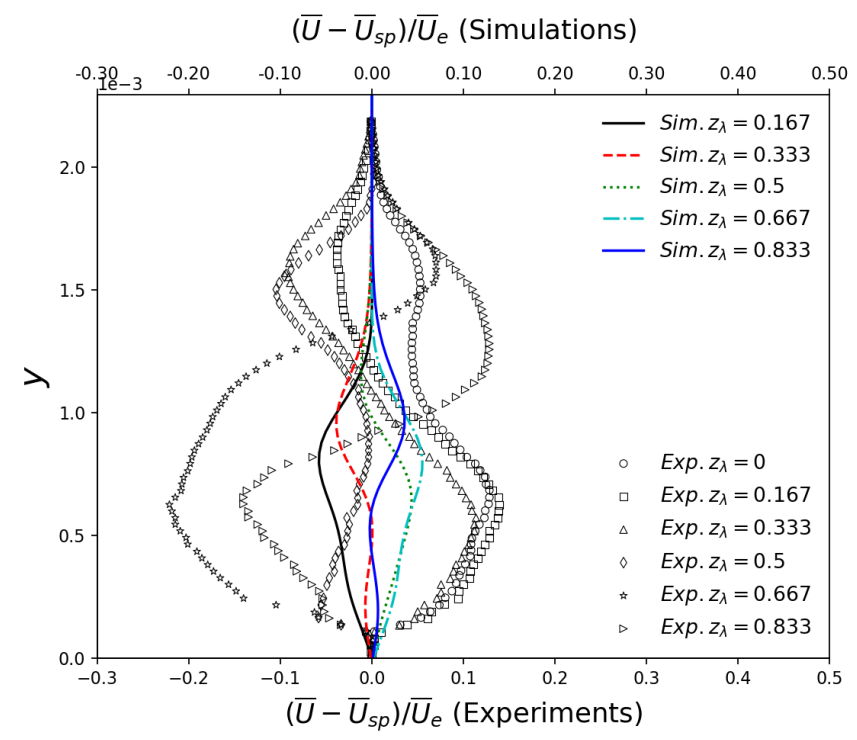

Fig. 11 Streamwise velocity disturbance due to the stationary crossflow per experiments [1] and simulations of the smooth case \#2.

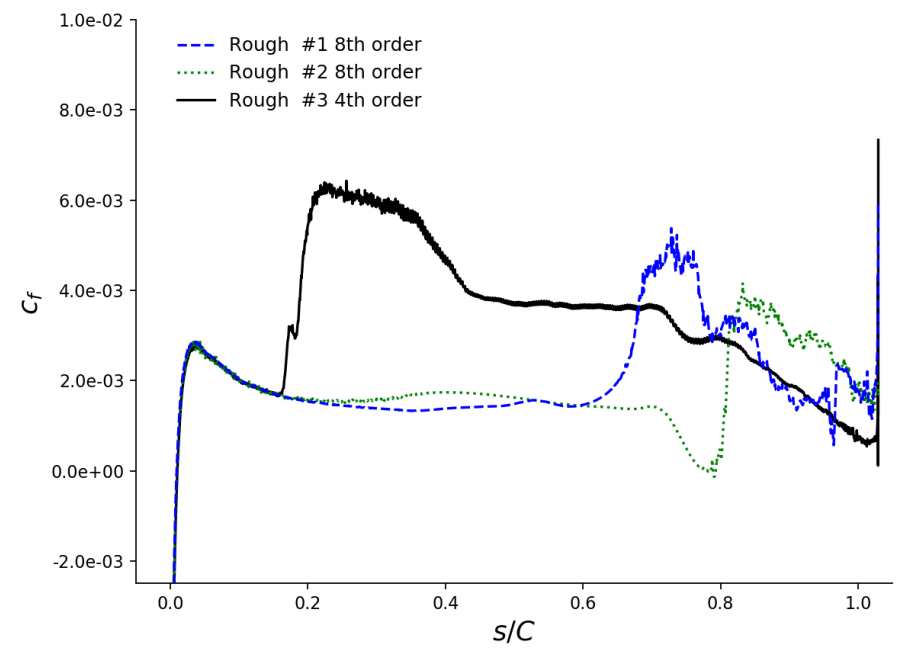

Fig. 12 Time-averaged friction coefficient on the upper side of the wings with leading edge roughness.

the computational resources available, and Gregory Matthews with InuTeq at NAS for attending to the large Skylake Elektra simulation jobs required for this work. Special thanks are extended to Karim Shariff in the Computational Physics Branch at NASA Ames for his guidance, and to Dirk Ekelschot with the Universities Space Research Association for helpful discussions.

\section{References}

[1] Dagenhart, J., and Saric, W., "Crossflow stability and transition experiments in swept-wing flow," Tech. Rep. TP-1999-209344, NASA, July 1999.

[2] Slotnick, J., Khodadoust, A., Alonso, J., Darmofal, D., Gropp, D., Lurie, E., and Mavriplis, D., "CFD vision 2030 study: a path to revolutionary computational aerosciences,” Tech. Rep. NASA/CR-2014-218178, CR-2014-218178, 2014. 


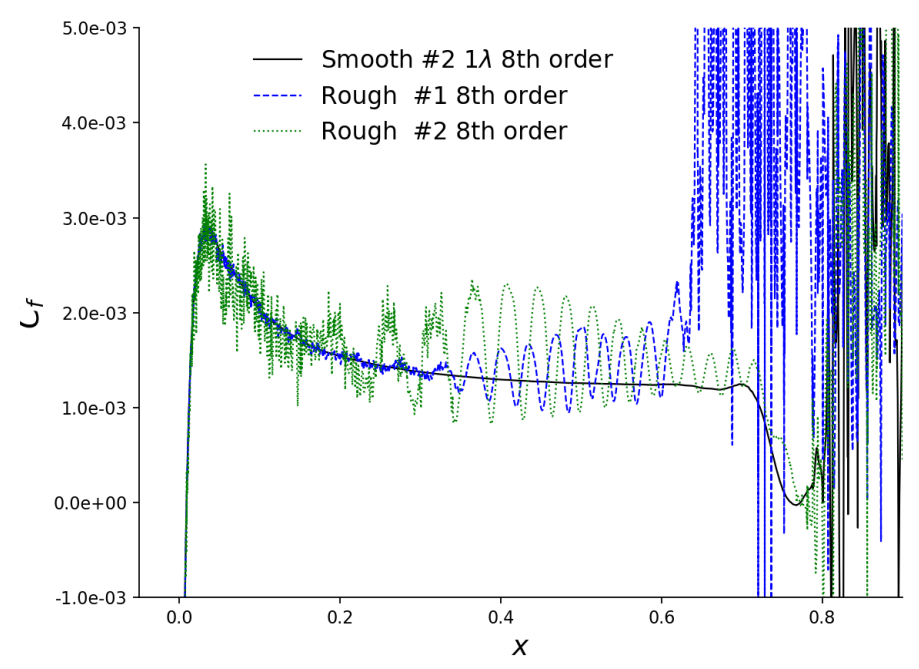

Fig. 13 Instantaneous coefficient on the upper side of the rough wing cases.

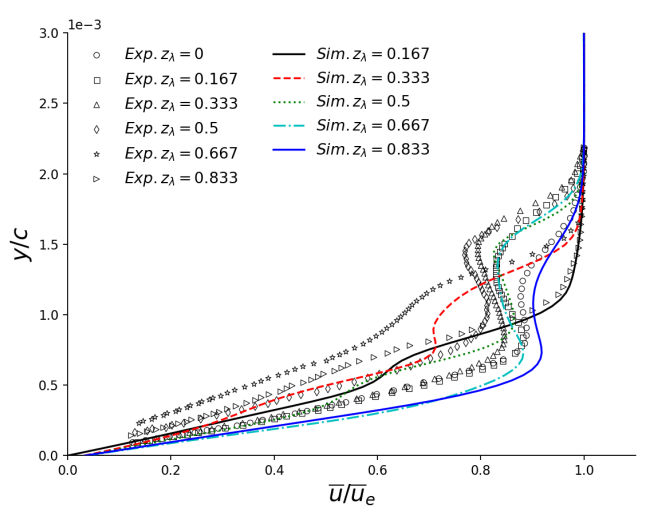

(a) $\bar{U} / U_{e}$

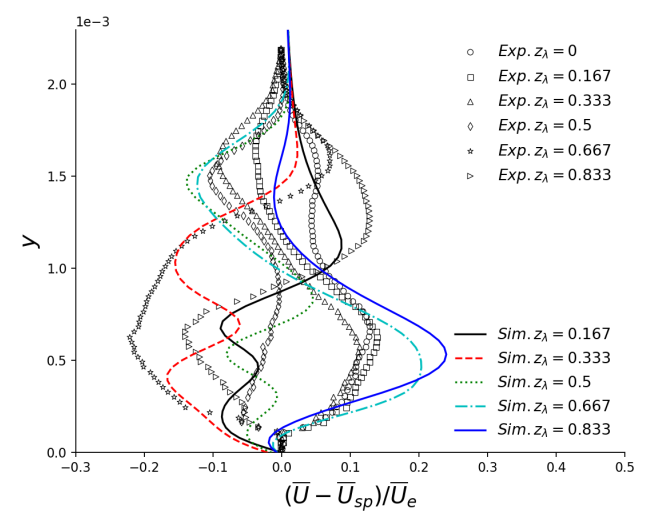

(b) Crossflow

Fig. 14 Velocity and crossflow perturbations along vertical cutlines at different stations along the wing sweep $(x / c=0.55)$ for the case of roughness \#1.

[3] Arthur, M., and Atkin, C., "Transition modelling for viscous flow prediction," 36th AIAA Fluid Dynamics Conference and Exhibit, 2006. doi:10.2514/6.2006-3052.

[4] Krumbein, A., Krimmelbein, N., and Schrauf, G., "Automatic Transition Prediction in Hybrid Flow Solver, Part 1: Methodology and sensitivities," Journal of Aircraft, Vol. 46, No. 4, 2009, pp. 1176-1190. doi:10.2514/1.39736.

[5] Langtry, R., and Menter, F., "Correlation-based transition modeling for unstructured parallelized fluid dynamics codes," AIAA Journal, Vol. 47, No. 12, 2009, pp. 2894-2906. doi:10.2514/1.42362.

[6] Krimmelbein, N., Krumbein, A., and Grabe, C., "Validation of transition modeling techniques for a simplified fuselage configuration,” Kissimmee, FL, 2018. doi:10.2514/6.2018-0030.

[7] Coder, J., Pulliam, T., and Jensen, J., "Contributions to HiLiftPW-3 using structured, overset grid methods," SciTech Forum, 2018. doi:10.2514/6.2018-1039.

[8] Ashton, N., Denison, M., and Zastawny, M., "3rd High-Lift Workshop summary paper - OpenFOAM, STAR-CCM+ \& LAVA simulations on unstructured grids,” 2018 AIAA Aerospace Sciences Meeting, 2018. doi:10.2514/6.2018-1253.

[9] Sørensen, N.N., Bechmann, A., and Zahle, F., “3D CFD computations of transitional flows using DES and a correlation based transition model," Wind Energy, Vol. 14, 2011, pp. 77-90. doi:10.1002/we.404. 


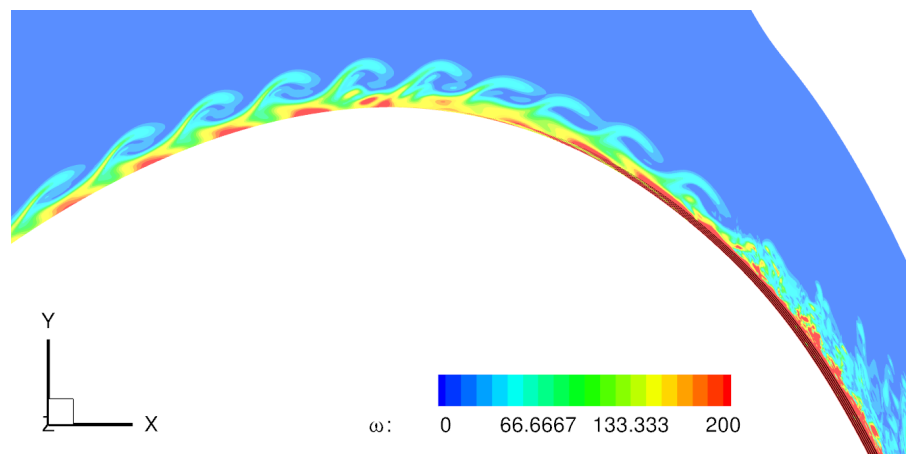

(a) $\mathrm{z}=0$ (y-direction scaled by a factor of 10 )

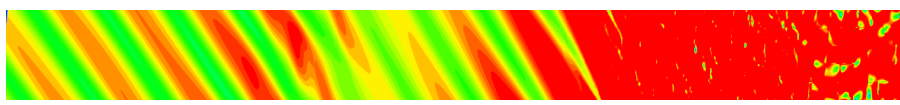

(b) top view (z-direction scaled by a factor of 10)

Fig. 15 Vorticity amplitude over the top surface of the wing for roughness case \#1.

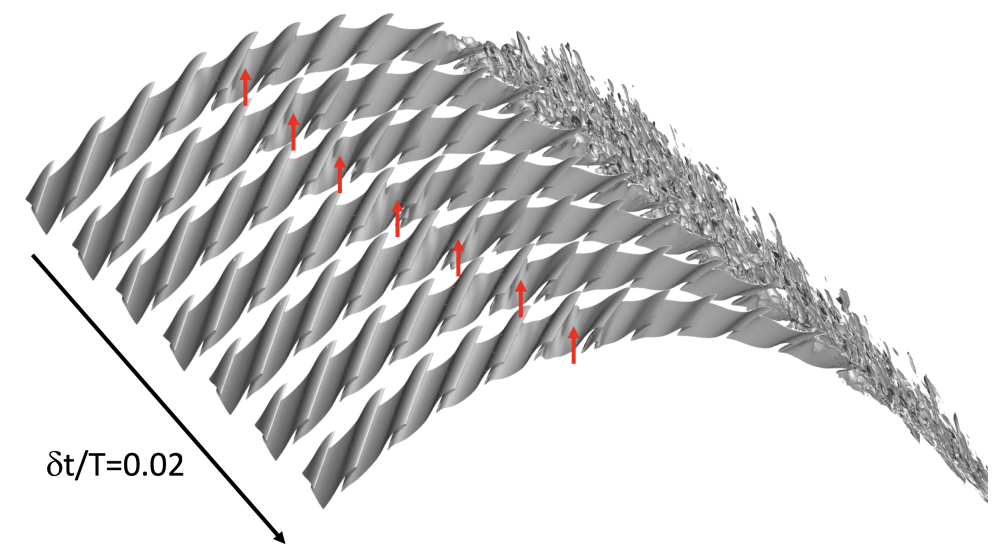

Fig. 16 Time evolution of the vorticity amplitude over the top surface of the wing with roughness \#1, in a region centered at $x / C=0.5$ (isocontour at $\omega=50$ ).

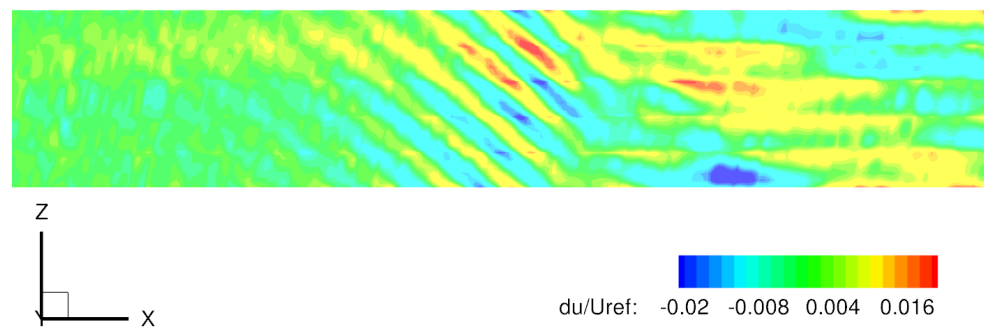

Fig. 17 Spanwise variation of streamwise velocity for the roughness case \#3, in a region close to the leading edge, centered around $x / C=0.15$ (z-direction scaled by a factor of 5 ).

[10] Booth, D., "A Comparison of Hybrid Reynolds-averaged Navier-Stokes/Large-Eddy Simulation (RANS/LES) and Unsteady RANS Predictions of Separated flow for a variable-speed power-turbine blade operating with low inlet turbulence levels," Tech. Rep. ARL-TR-8191, US Army Research Laboratory, October 2017. 


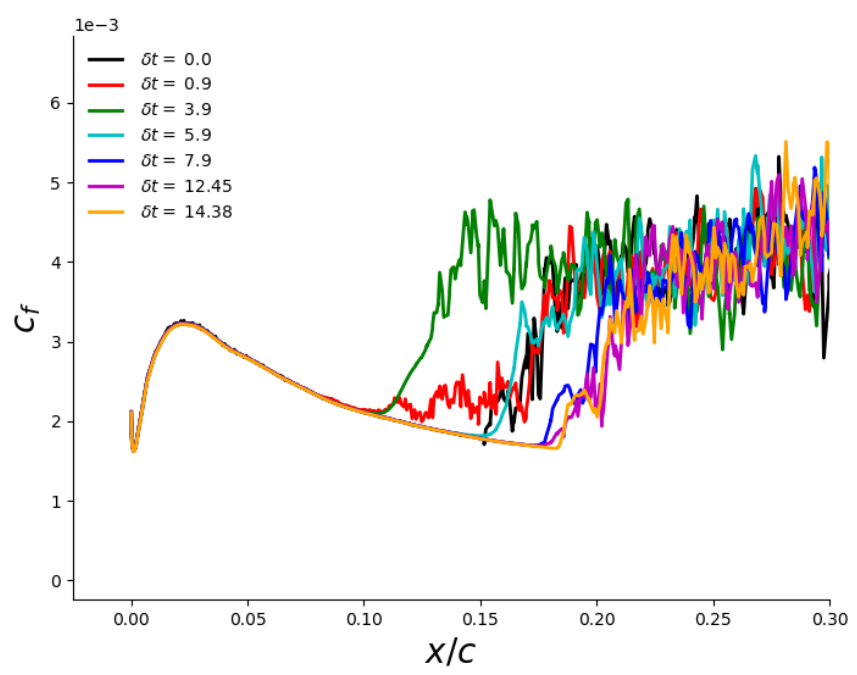

Fig. 18 Time evolution of the span-averaged friction coefficient for the roughness case \#3, after the surface roughness was switched off.

[11] Coder, J., and Ortiz-Melendez, H., "Transitional Delayed Detached Eddy Simulation of multielement, high-lift airfoils," 2018 Applied Aerodynamics Conference, AIAA AVIATION Forum, 2018. doi:10.2514/6.2018-2846.

[12] Seyfert, C., and Krumbein, A., "Correlation-based transition transport modeling for three-dimensional aerodynamic configurations," 50th AIAA Aerospace Sciences Meeting, 2012. doi:10.2514/6.2012-448.

[13] Medida, S., and Baeder, J., "A new crossflow transition onset criterion for RANS turbulence models," 21st AIAA Computational Fluid Dynamics Conference, 2013. doi:10.2514/6.2013-3081.

[14] Grabe, C., and Krumbein, A., "Extension of the gamma-Rethetat model for prediction of crossflow transition," 52nd Aerospace Sciences Meeting, 2014. doi:10.2514/6.2014-1269.

[15] Langtry, R., Sengupta, K., Yeh, D., and Dorgan, A., "Extending the gamma-Rethetat local correlation based transition model for crossflow effects," 45th AIAA Fluid Dynamics Conference, 2015. doi:10.2514/6.2015-2474.

[16] Grabe, C., Nie, S., and Krumbein, A., "Transition transport modeling for the prediction of crossflow transition," 34th AIAA Applied Aerodynamics Conference, 2016. doi:10.2514/6.2016-3572.

[17] Radeztsky Jr., R., Reibert, M., and Saric, W., "Effect of micron-sized roughness on transition in swept-wing flows," $31 s t$ Aerospace Sciences Meeting and Exhibit, Reno, NV, 2013. doi:doi.org/10.2514/6.1993-76.

[18] Kreplin, H.-P., Vollmers, H., and Meier, H., "Wall shear stress measurements on an inclined prolate spheroid in the DFVLR 3 m x 3 m low speed wind tunnel, Göttingen,” Tech. Rep. IB 222-84 A 33, DFVLR-AVA, 1985.

[19] Saric, W., Reed, H., and White, E., "Stability and transition of three-dimensional boundary layer," Annual Review of Fluid Mechanics, Vol. 35, 2003, pp. 413-440. doi:10.1146/annurev.fluid.35.101101.161045.

[20] Diosady, L., and Murman, S., "Design of a variational multiscale method for turbulent compressible flows," 21st AIAA Computational Fluid Dynamics Conference, San Diego, CA, 2013. doi:10.2514/6.2013-2870.

[21] Diosady, L., and Murman, S., "Higher-order methods for compressible turbulent flows using entropy variables," 53rd AIAA Aerospace Sciences Meeting, Kissimmee, FL, 2015. doi:10.2514/6.2015-0294.

[22] de Wiart, C., Diosady, L., Garai, A., Burgess, N., Blonigan, P., Ekelschot, D., and Murman, S., "Design of a modular monolithic implicit solver for multi-physics applications," 2018 AIAA Aerospace Sciences Meeting, Kissimmee, FL, 2018. doi:10.2514/6.2018-1400.

[23] Murman, S., Diosady, L., Garai, A., and Ceze, M., “A space-time discontinuous-Galerkin approach for separated flows," 54th AIAA Aerospace Sciences Meeting, San Diego, CA, 2016. doi:10.2514/6.2016-1059. 
[24] Diosady, L., and Murman, S., "A linear-elasticity solver for higher-order space-time mesh deformation," 2018 AIAA Aerospace Sciences Meeting, Kissimmee, FL, 2018. doi:10.2514/6.2018-0919.

[25] Ismail, F., and Roe, P., "Affordable, entropy-consistent Euler flux functions II: entropy production at shocks," Journal of Computational Physics, Vol. 228, 2009, pp. 5410-5436. doi:10.1016/j.jcp.2009.04.021.

[26] Bassi, F., and Rebay, S., Lecture Notes in Computational Science and Engineering, Springer, Berlin, Heidelberg, Germany, 2000, Vol. 11, Chaps. GMRES discontinuous Galerkin solution of the compressible Navier-Stokes equations, pp. 197-208. doi:10.1007/978-3-642-59721-3_14.

[27] Ceze, M., Diosady, L., and Murman, S., "Development of a high-order space-time matrix-free adjoint solver," 54th AIAA Aerospace Sciences Meeting, San Diego, CA, 2016. doi:10.2514/6.2016-0833.

[28] Diosady, L., and Murman, S., "DNS of flows over periodic hills using a discontinuous Galerkin spectral-element method," 44th AIAA Fluid Dynamics Conference, Atlanta, GA, 2014. doi:10.2514/6.2014-2784.

[29] Garai, A., Diosady, L., Murman, S., and Madavan, N., "Scale-resolving simulations of low-pressure turbine cascades with wall roughness using a spectral-element method," ASME Turbo Exp 2018: Turbomachinery Technical Conference and Exposition, Oslo, Norway, 2018. doi:10.1115/GT2018-75982. 